TITLE:

\title{
Forward and backward laser- guided motion of an oil droplet
}

$\operatorname{AUTHOR}(S)$ :

Rybalko, S; Magome, N; Yoshikawa, K

CITATION:

Rybalko, S ... [et al]. Forward and backward laser-guided motion of an oil droplet. Physical Review E 2004, 70(4): 046301.

ISSUE DATE:

2004-10

URL:

http://hdl.handle.net/2433/49860

RIGHT:

Copyright 2004 American Physical Society 
PHYSICAL REVIEW E 70, 046301 (2004)

\title{
Forward and backward laser-guided motion of an oil droplet
}

\author{
Sergei Rybalko, ${ }^{*}$ Nobuyuki Magome, and Kenichi Yoshikawa \\ Department of Physics, Graduate School of Science, Kyoto University \& CREST, Kyoto 606-8502, Japan
}

(Received 26 March 2004; published 5 October 2004)

\begin{abstract}
Directed motion of an oil droplet floating in an aqueous solution is generated by using a laser beam. Interestingly, the direction of the droplet motion can be switched between forward and backward by changing the optical path of the laser through the droplet. This motion is caused above a certain critical power of the laser, and above this value the velocity increases almost linearly with the power. The mechanism of this directed motion is explained as follows: the oil droplet is locally heated by a narrow laser beam, this local heating induces a specific mode of convection inside the droplet, and this generated convective motion produces translational directed motion of the droplet.
\end{abstract}

DOI: 10.1103/PhysRevE.70.046301

PACS number(s): 47.20.Dr, 44.25.+f, 47.20.Bp, 47.27.Te

It has been well established that a laser exerts two different kinds of force on an object; optical pressure and attraction toward the focus, i.e., laser trapping (see, e.g., Refs. [1-3]). For objects floating in the gas phase, it has been found that some types of objects move toward the source of the laser beam, which has been described in terms of radiometric force (see, e.g., Ref. [4], and references therein). However, as far as we know, there has been no report on the controlled directed forward and backward motion of an object solely due to a change in the position of the illumination of a laser on the object. In the present report, we describe a methodology for the directed motion of an oil droplet in an aqueous solution that is generated by a laser beam. Significantly, the direction of the droplet motion can be switched between forward and backward depending on the optical path of the laser through the droplet. This controlled motion can be explained in terms of photomechanical energy conversion based on a type of mechanism: an oil droplet is locally heated by a narrow laser beam, this local heating induces convection inside the droplet, and this convective motion produces translational directed motion of the droplet. The intrinsic mechanism of photomechanical energy transduction has been a longstanding problem in natural science and technology $[5,6]$. Over the past few years, some studies on complex convection in liquids driven by a laser have been reported [7]. Convection in fluids has been a fruitful system for the study of nonlinear, nonequilibrium patterns for more than 100 years [8-10]. Many years passed before it was conclusively shown that surface tension gradients, or Marangoni forces, were crucial [11,12]. Local strong heating of a free surface between either liquid and air or two liquids can also generate well-known Marangoni, or thermocapillary, convection in liquids (see, e.g., Ref. [13], and references therein). Experiments on laser-induced Marangoni convection have been performed under various conditions: in microgravity environments to minimize the influence of buoyancy effects [14-16], on the spreading of microscale liquid films over solid substrate to probe and to control dynamics of the flow inside [17], and on trapping the gas bubbles during a liquid/

*Email address: rybalko@chem.scphys.kyoto-u.ac.jp gas phase separation [18-20]. In addition, laser-induced Marangoni convection is of interest in the welding industry since it can affect the weld pool shape, and the consequent production of ripples during laser welding may cause significant roughness of the surface of the treated materials $[21,22]$. At present, local very strong heating of a surface can be easily achieved by high-power lasers, and therefore it is easy to induce thermocapillary convection. If a system is small but macroscopic, such as an oil droplet in water, this convection may produce mechanical work. As a result, macroscopic mechanical motion is expected to be generated in a small liquid system by laser light.

In the present study, we performed experiments on a nitrobenzene oil droplet in a water solution under strong laser irradiation. Decreasing the interfacial tension between water and air by adding SDS (sodium dodecyl sulfate, $0.5 \mathrm{mM}$ ) allowed us make the droplet assume a hemispherelike shape and to float just under the surface of the water-air interface. The floating droplet is approximately $5 \mathrm{~mm}$ in diameter and $5 \mathrm{~mm}$ thick. We used a continuous-wave laser with a wavelength of $532 \mathrm{~nm}$. The power of the laser can be varied from $0.1 \mathrm{~W}$ to $1.0 \mathrm{~W}$. To produce the maximum effect in local heating, the laser beam was narrowed by a lens to $0.6 \mathrm{~mm}$ in diameter. To achieve very high absorbance of the light inside the oil, we changed the color of the oil by adding $\mathrm{I}_{2}(5 \mathrm{mM})$ and KI (until saturation at room temperature) so that the droplet was then tinted brown or red. For comparison, we measured the absorbance of both tinted and untinted oil. The absorption coefficient increased from 0.1 (untinted oil) to 2.7 (tinted oil) at $532 \mathrm{~nm}$.

Figure 1 shows the floating geometry of a droplet in an elongated rectangular glass vessel. All of the container walls are made of 1-mm-thick Plexiglas. The laser beam is aimed at the oil droplet from the left-hand side. The oil moves from the left to right or vice versa depending on the laser position: backward motion is seen if the laser is near the air-water interface and forward motion occurs if the laser is directed at the bottom of the droplet.

For greater detail, in Fig. 2(a) we show a spatiotemporal plot of the oil droplet movement throughout the whole experiment. The snapshots were prepared using a CCD camera attached to the front wall of the vessel. We occasionally 


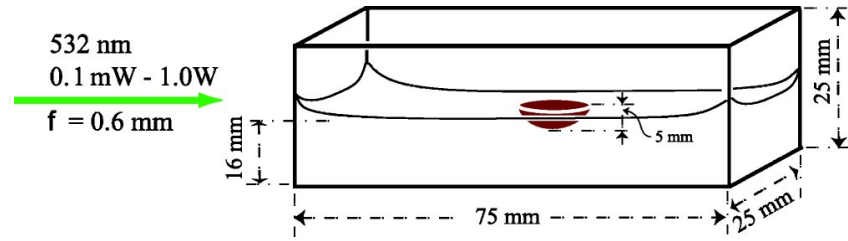

FIG. 1. Schematic diagram of the experiment: a c.w. laser beam $(\lambda=532 \mathrm{~nm}$, Millenia, Spectra Physics) irradiates the droplet (approximately $5 \mathrm{~mm}$ in diameter) from the left side. The droplet moves from the left to right or vice versa depending on the position of the laser. The power and radius of the laser are shown on the arrow.

switched between the two different types of motion by changing the position of the laser to demonstrate the repeatability of this phenomenon.

If the laser is used to irradiate the surface [Fig. 2(a), white ranges on the time scale], a significant portion of the light energy is absorbed at transmission of the laser beam through the droplet of oil. In this case, the layer of oil that is heated locally is almost straight line, i.e., the diameter of crosssection of the droplet at the air-water interface. Energy ab- sorption is enhanced when the oil is tinted with iodine. As the light beam passes through the oil, its intensity decreases exponentially. This leads to a temperature gradient along the layer mentioned above. Strong local heating of oil results in complex convection inside the droplet and forces it finally to move beside the laser (backward motion). Under the other condition, i.e., with irradiation at the bottom of the oil droplet [Fig. 2(a), gray ranges on the time scale], the beam of the laser refracts on the oil-water interface and most of the light energy is absorbed in the refracted laser beam, i.e., in a thin layer along a line from the bottom of the droplet to its far right-hand side. Due to the high absorption coefficient, there is a temperature gradient along the refracted beam that results in local nonuniform oil heating. This in turn causes complex convective flow in the bulk of the oil. As a result, the droplet moves away from the laser (forward motion).

We tried to measure the velocity profile in a droplet under laser irradiation at a specific position as a function of the laser power. We conducted systematic experiments to document the movement of the oil droplet and measured the resulting velocity and the maximal temperature with changes in the laser power, which was varied from 0.1 to $1.0 \mathrm{~W}$ in steps of $0.1 \mathrm{~W}$. Figure 2(b) shows the change in droplet

(a)

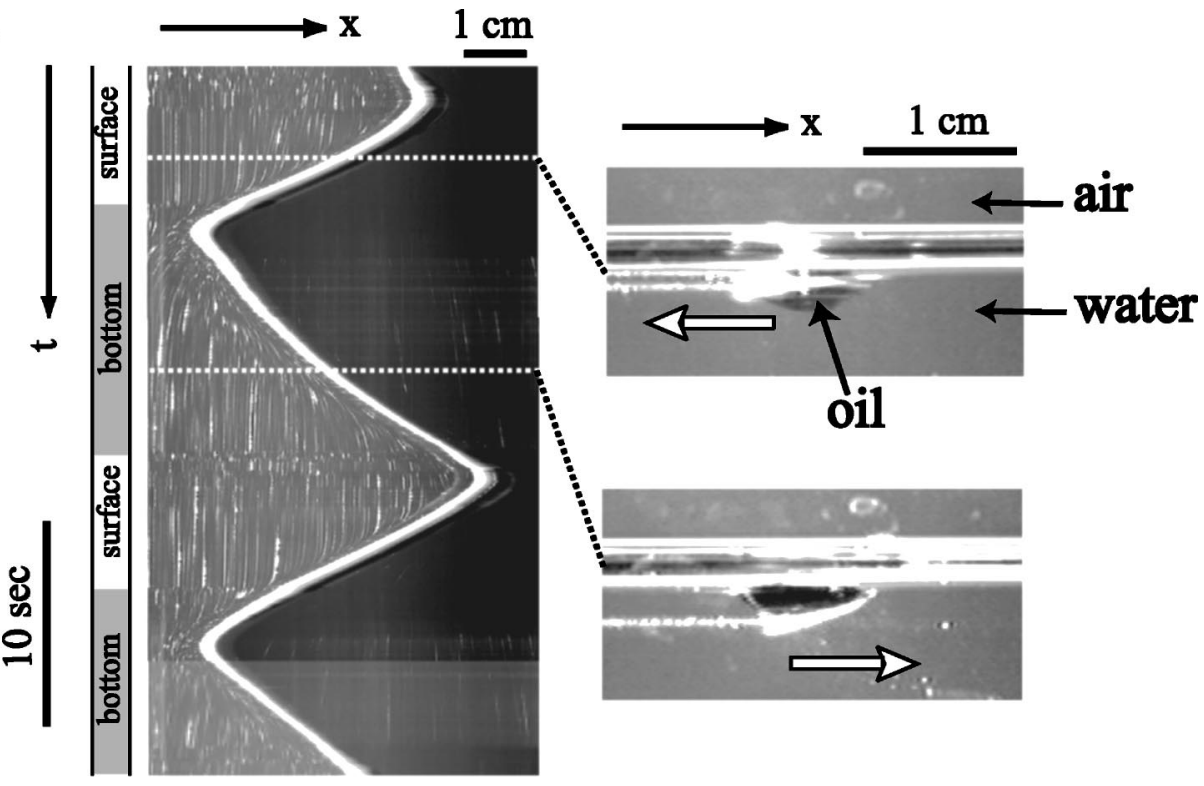

(b)

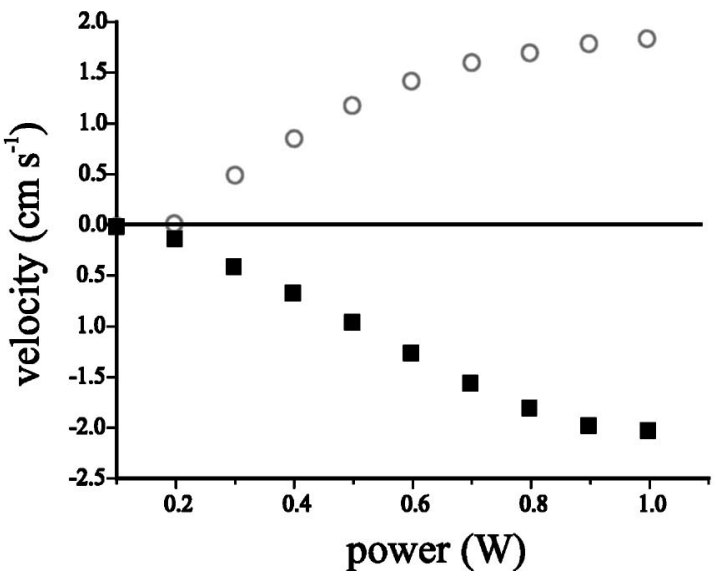

FIG. 2. (a) Spatiotemporal images of droplet motion constructed from time-successive video frames. The horizontal axis in the left figure is the cross-section parallel to the laser beam. The white trace in the figure indicates the position of the oil droplet. The location of laser irradiation, either upper surface or bottom of the droplet, is indicated on the left time bar. The pictures on the right are snapshots. (b) Dependence of the velocity of the droplet on the laser power. The open circles (positive values) represent forward movement of the droplet (laser at the bottom) and the solid squares (negative values) denote backward movement (laser near the surface). 
(a)

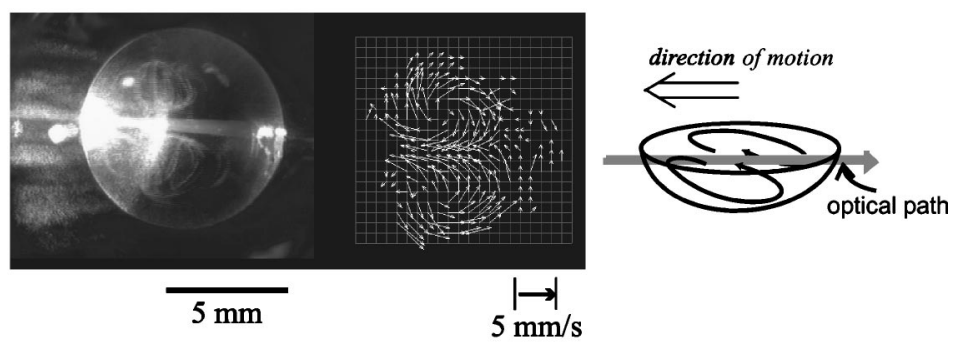

(b)

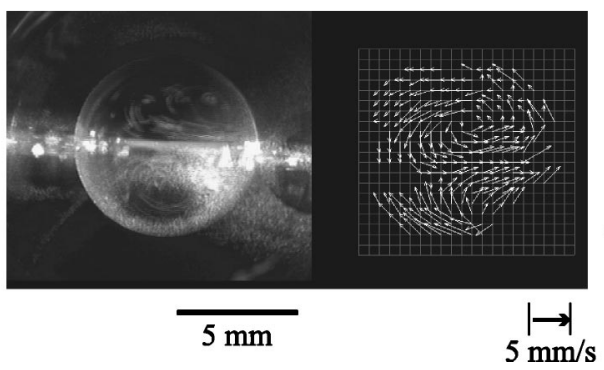

FIG. 3. Convective flow in an oil droplet placed in a narrow cylindrical test tube: The laser beam is focused at the oil-air interface (a) and at the bottom of the oil droplet (b). Left column: snapshots taken from above with the contrast adjusted to visualize the generated flow. Middle column: vector field of convective current reconstructed from a movie of the experiment using image-analysis software ("Flow Vec," Library). Right column: schematic drawing of 3D convection made from data based on real observations and clips from top- and side-view cameras. velocity with an increase in the laser power. The velocity of the droplet was measured by analyzing recorded images. The plot of velocity [Fig. 2(b)] has horizontal asymptotes for both forward motion (positive values) and backward motion (negative values). Using a noncontact IR thermometer (IT340 , Horiba), we found that the temperature of the oil droplet increases with an increase in the laser power; the temperature at the hottest region is $45^{\circ} \mathrm{C}$ at $P=0.5 \mathrm{~W}$ and $50{ }^{\circ} \mathrm{C}$ at $P$ $=1.0 \mathrm{~W}$ [23].

The key point of these experiments on the capture of an oil droplet by a laser beam is that local heating of the oil leads the system far from equilibrium, and as a result to complex convection inside the droplet. For a detailed analysis of convective flow in oil, we conducted some experiments in which we irradiated a droplet in a narrow cylindrical vertical vessel (a test tube). The conditions in this experiment do not allow the droplet to move due to the large meniscus, and thus it is easier to examine convective currents.

Figure 3 shows pictures of oil droplets under laser irradiation near the surface (a) and at the bottom (b). They show convective vortices in the top-view projection (left column), a vector field of convective currents (middle column) and a schematic drawing of 3D convection (right column) made from movie clips taken from top- and side-view cameras. To visualize the motion of oil, polystyrene micro-beads with an average diameter of $0.25 \mathrm{~mm}$ were used. Under both conditions, it is clear that vortices exist in two dimensions. Due to the hemispherelike form of the droplet, complex threedimensional convection is also thought to be involved in this process. By analyzing this convection in more detail using recorded data, we found that it is a result of two main components. First, strong local heating causes gravitational or free convection in the oil driven by a density gradient that leads to the appearance of buoyancy forces. This well-known phenomenon induces vertical circular movement. In addition, the most important effect in this experiment is the presence of a thermocapillary (or Marangoni) convection-driven surface tension gradient. Under free surface heating, surface tension effects are included to the extent that local shear stresses are generated by variation of the surface tension due to the thermal gradient at the oil-water interface. These surface tension gradients could induce the convection of oil in a horizontal plane. The presence of viscosity (or friction) between oil and water leads to interfacial forces between oil and water. The viscosity between oil and water is greater than that between oil and air. Convective currents near the interface between oil and water mainly flow in one direction. As a result, the total interfacial force between oil and water is greater than that between oil and air at the surface. This leads to movement of the droplet in a certain direction. By changing the position of the laser, we change the layer of oil that is locally heated. This changes the geometry of complex convection [Fig. 3 (right column)] and reverses the direction of the oil droplet movement.

Moreover, it should be mentioned that the behavior of a droplet is very sensitive to its radius. In fact, if the radius of a droplet is small enough, no convection is generated in the oil. And vice versa, a large droplet is too heavy and does not move, or cannot float and falls down at the bottom of a vessel. Thus, the diameter of a droplet in our experiments (approximately $5 \mathrm{~mm}$ ) was chosen optimal to demonstrate the described phenomenon.

With regard to the generation of vectorial motion under nonequilibrium, previous reports have described oil-water systems in which chemical energy is converted into mechanical work [24-26]. These reports have demonstrated movement in an oil-water system induced by chemically driven Marangoni instability. The oscillation and self-movement of the interface have been suggested to be attributed to the periodic formation and destruction of a surfactant monolayer at the interface. In one study [26], a rhythmic change in interfacial tension was driven by a rhythmic change in the Belousov-Zhabotinsky reaction that was generated in a small droplet of chemical active media [26]. Thus, it induced directional Marangoni convective motion in the chemical droplet and led to droplet movement.

In summary, we have developed an experimental fluid system that demonstrates the photomechanical conversion of energy. The system consists of a nitrobenzene oil droplet (about $5 \mathrm{~mm}$ in diameter) in a water solution. We changed the color of the oil droplet by adding iodine and irradiated it 
with a laser that had been narrowed by a lens. We found that the direction of macroscopic droplet motion depended only on the position of the laser. This experimental system is easy to implement (Fig. 1), due to its simple geometry, and the observed phenomenon is very reproducible. By conducting many experiments in both long and tall, narrow vessels, we found that the main cause of this energy transformation is complex 3D convective motion inside the oil droplet, or coupled thermocapillary and gravitational convection, caused by strong local heating of the oil-water interface. The internal convective dynamics could be directly observed (Fig. 3) when very small inactive microbeads were placed in the bulk of the oil. The presence of viscosity between oil and water leads to interfacial forces between oil and water, and as a result to macroscopic oil droplet motion. We expect that this complex photomechanical phenomenon can be very well described by Navier-Stokes equations with a Boussinesq approximation, which are often used to investigate both gravitational and thermocapillary convection. Note that the system is hemispherical and thus requires the use of curvilinear coordinates for an adequate description. Some theoretical interpretations will be developed and the resulting model will be subjected to a numerical analysis in a future study.

This research was supported in part by a Grant-in-Aid from the Ministry of Education, Science, Sports and Culture of Japan, by "Ground-based Research Announcement for Space Utilization" promoted by the Japan Space Forum, and by a Grant-in-Aid for the 21st Century COE "Center for Diversity and Universality in Physics."
[1] A. Ashkin, J. M. Dziedzic, J. E. Bjorkholm, and S. Chu, Opt. Lett. 11, 288 (1986).

[2] S. Chu, Science 253, 861 (1991).

[3] T. Harada and K. Yoshikawa, Appl. Phys. Lett. 81, 4850 (2002).

[4] P. C. Ke and M. Gu, Appl. Opt. 38, 160 (1999).

[5] H. Monjoshiro, M. Tanaka, and H. Watarai, Chem. Lett. 32, 254 (2003).

[6] H. Monjoshiro, M. Tanaka, and H. Watarai, Chem. Lett. 32, 788 (2002).

[7] M. Guglioti, M. S. Baptista, and M. J. Politi, Langmuir 18, 9792 (2002).

[8] H. Bénard, Rev. Gen. Sci. Pures Appl. 11, 1261 (1900).

[9] C. Normand and Y. Pomeau, Rev. Mod. Phys. 49, 581 (1977).

[10] M. C. Cross and P. C. Hohenberg, Rev. Mod. Phys. 65, 851 (1993).

[11] M. J. Block, Nature (London) 178, 650 (1956).

[12] J. R. A. Pearson, J. Fluid Mech. 4, 489 (1958).

[13] M. F. Schatz and G. P. Neitzel, Annu. Rev. Fluid Mech. 33, 93 (2001).

[14] Y. Kamotani, S. Ostrach, and A. Pline, Phys. Fluids 6, 3601 (1994).

[15] J. P. Longtin, K. Hijikata, and K. Ogawa, Int. J. Heat Mass Transfer 42, 85 (1999).

[16] K. Ohsaka, S. S. Sadhal, and A. Rednikov, J. Heat Transfer 124, 599 (2002).
[17] N. Garnier, R. O. Grigoriev, and M. F. Schatz, Phys. Rev. Lett. 91, 054501 (2003).

[18] D. Beysens et al., Europhys. Lett. 59, 245 (2002).

[19] V. Y. Bazhenov et al., Appl. Phys. B: Photophys. Laser Chem. 49, 485 (1989).

[20] A. Marcano et al., Appl. Phys. B: Photophys. Laser Chem. 56, 343 (1993).

[21] C. Limmaneevichitr and S. Kou, Weld. J. (Miami, FL, U. S.) 79, 231S (2000).

[22] C. Limmaneevichitr and S. Kou, Weld. J. (Miami, FL, U. S.) 79, $126 \mathrm{~S}$ (2000).

[23] The dependence of the increase in temperature on the laser power is thought to be approximated by the linear equation $\Delta T=K P$, where $K$ is some constant that is directly proportional to the absorbance of oil, the geometry of the droplet and the experimental conditions (some form-factor), and inversely proportional to the heat conductivity of oil. For more details and some discussion, see S. Wurlizer, C. Lautz, M. Liley, C. Duschl, and T. M. Fischer, J. Phys. Chem. B 105, 182 (2001).

[24] K. Yoshikawa and N. Magome, Bull. Chem. Soc. Jpn. 66, 3352 (1993).

[25] N. Magome and K. Yoshikawa, J. Phys. Chem. 100, 19102 (1996).

[26] H. Kitahata, R. Aihara, N. Magome, and K. Yoshikawa, J. Chem. Phys. 116, 5666 (2002). 